\title{
Adult endothelial progenitor cells from human peripheral blood maintain monocyte/macrophage function throughout in vitro culture
}

\author{
Shi Ju Zhang ${ }^{1}$, Hao Zhang ${ }^{1}$, Ying Jie Wei ${ }^{1}$, Wen Jun Su${ }^{1}$, Zhong Kai Liao ${ }^{1}$, Mai Hou ${ }^{1}$, Jian Ye Zhou ${ }^{1}$, \\ Sheng Shou $\mathrm{Hu}^{1}$ \\ ${ }^{1}$ Research Center for Cardiovascular Regenerative Medicine, Ministry of Health, and Department of Cardiovascular Surgery, Car- \\ diovascular Institute and Fu-Wai Hospital, PUMC and CAMS, 167 Beilishi Road, West District, Beijing 100037, China
}

Mononuclear cells (MNCs) isolated from peripheral blood by density gradient centrifugation were plated on human fibronectin-coated culture plates and cultured in EGM-2 medium. Attached spindle-shaped cells, reported as endothelial progenitor cells (EPCs) by some investigators, had elongated from adherent round cells, but had not proliferated from a small number of cells as supposed previously. The growth curve of the primary EPCs showed that the cells had little proliferative capacity. Flow cytometry analysis showed that the cells could express some of the endothelial lineage markers, while they could also express CD14, which is considered a marker of monocyte/macrophage lineages throughout culture. In endothelial function assays, the cells demonstrated a lower level of expression of eNOS than mature endothelial cells in the reverse transcription-polymerase chain reaction and did not show an ability to develop tube-like structures in angiogenesis assay in vitro. In this study, we identified the monocytoid function of EPCs by the combined Dil-labeled acetylated low-density lipoprotein (Dil-Ac-LDL) and Indian ink uptake tests. All the cells were double positive for DilAc-LDL and Indian ink uptake at days 4, 14 and 28 of culture, which means the EPCs maintained monocytoid function throughout the culture. Therefore, although adult EPCs from peripheral MNCs have some endothelial lineage properties, they maintain typical monocytic function and have little proliferative capacity.

Cell Research (2006) 16:577-584. doi:10.1038/sj.cr.7310075; published online 15 June 2006

Keywords: mononuclear cells, endothelial progenitor cells, endothelial cells, monocyte/macrophages

\section{Introduction}

It was thought that endothelial progenitor cells (EPCs) and vasculogenesis, which are involved in the development of the circulation system in the embryonic stage, do not exist in the adult body $[1,2]$. However, due to the discovery of adult EPCs in 1997 [3], this dogma has been challenged and researchers have come to believe that EPC transplantation may augment vasculogenesis, which has a more significant therapeutic effect on ischemic disease than angiogenesis. Some researchers have applied the EPCs for this purpose and acquired beneficial results [4-10]. Since,

Correspondence: Sheng Shou Hu

Tel: +86-10-68334788; Fax: +86-10-68313012;

E-mail:huss@163bj.com or drzhanghao@yahoo.com.

Received 21 Jul 2005; revised 5 Feb2006; accepted 16 Feb 2006; published online 15 Jun 2006 according to Asahara et al. [3], EPCs have a significant proliferation capacity and can differentiate into mature endothelial cells (ECs), it is not surprising that they have been used as a substitute for ECs in tissue engineering research $[11,12]$. Encouraged by these promising studies, more and more researchers are studying the practical application of EPCs.

However, recent disputes on EPCs led to the further investigation of their biological characteristics. CD14positive cells, which are typically CD34-negative, have also been suggested to be the source of EPCs, according to Harraz et al. [13]. Nakul-Aquaronne et al. [14] reported that CD34-positive cells, of which some are EPCs according to Asahara et al. [3], differentiate along the monocyte/ macrophage lineage in vitro. Therefore, the exact origin of EPCs is not clear, leading to the hypothesis that there may be two types of EPCs: one type can coexpress monocyte/ macrophage and EC markers and the other has a powerful 
capacity for proliferation and can only express typical EC markers [15-17]. In this regard, the culture of preselected mononuclear cells (MNCs) may preclude certain types of EPCs due to the uncertainty of the origin.

The culture of unprocessed MNCs is another most commonly used method to obtain EPCs [18-20], because of the low expenditure and simple procedure, especially when the culture of cells is for practical application. Meanwhile, since adherence to the culture plate is an intrinsic property of EPCs, this method may have another advantage, namely, we can obtain all EPCs regardless of the subset. In this study, we harvested all the adherent cells and examined their biological characteristics, especially from a functional point of view, to clarify the controversy about EPCs.

\section{Materials and Methods}

\section{Cell culture}

MNCs were isolated from $100 \mathrm{ml}$ of peripheral blood anticoagulated with heparin from healthy young volunteers (mean age $23.05 \pm 4.16$ years) by density gradient centrifugation over histopaque$10771(1.077 \mathrm{~g} / \mathrm{ml}$, Sigma) according to the manufacturer's protocol. The residual Ficoll in the solution containing the MNCs was washed away with PBS, and the cells were then adjusted to a density of 1 $\times 10^{6}$ cells $/ \mathrm{ml}$ in medium EGM-2, i.e., basal medium containing vascular endothelial growth factor (VEGF), basic fibroblast growth factor, epidermal growth factor, insulin-like growth factor-1, hydrocortisone, heparin and ascorbic acid (Clonetics) supplemented with $5 \%$ fetal calf serum (Gibco-BRL). The cells were then plated on human fibronectin-coated tissue culture chambers and cultured at $37{ }^{\circ} \mathrm{C}$ with $5 \% \mathrm{CO}_{2}$ in a humidified atmosphere. The medium was changed every 3 days.

All procedures followed were in accordance with institutional guidelines and the principles outlined in the Declaration of Helsinki.

\section{Proliferative capacity assay}

Growth curve MNCs were seeded in 24-well plates $\left(1 \times 10^{6} \mathrm{MNCs} /\right.$ well). From day 3 of culture, namely, the day of the first media change, 10 fields $(\times 100)$ were chosen randomly under an inverted microscope and pictures were taken. To avoid counting errors, the pictures were opened with Photoshop software, in which the cells that are counted can be marked. The mean number of cells was calculated and expressed as means \pm S.D. and was used for the description of the growth curve. The above-mentioned procedure was repeated every 3 days for 3 weeks. Four blood samples from different individuals were included in this assay.

BrdU incorporation assay Sterilized slides were put into culture wells and MNCs were then seeded onto the slide in the well. After 7 and 21 days of culture, $10 \mu \mathrm{l}$ of BrdU solution (BrdU, $50 \mathrm{mg}$; dimethylsulfoxide, $0.8 \mathrm{ml}$; water, $1.2 \mathrm{ml}$ ) was added to the well and incubated with the cells for $24 \mathrm{~h}$. Then, the cells on the slide were fixed with $4 \%$ polyformaldehyde and endogenous peroxidase was blocked using $3 \% \mathrm{H}_{2} \mathrm{O}_{2}$ for $10 \mathrm{~min}$ at $37^{\circ} \mathrm{C}$ and $2 \mathrm{~N}$ of $\mathrm{HCl}$ for 30 min at $20^{\circ} \mathrm{C}$. After two washes in PBS, the cells were permeabilized with $0.2 \%$ Triton X-100 in PBS for 10 min. After rinsing with PBS three times and blocking with goat serum for $15 \mathrm{~min}$, the slides with cells were then incubated with mouse antibodies against BrdU for $2 \mathrm{~h}$ at $37^{\circ} \mathrm{C}$. Negative controls were incubated in PBS (without the primary antibodies) under the same conditions. The slides were rinsed with PBS three times and then incubated with peroxidase-conjugated goat anti-mouse antibody at $37{ }^{\circ} \mathrm{C}$ for $45 \mathrm{~min}$. Finally, the slides were immersed in AEC staining solution (Sigma) for $30 \mathrm{~s}$. After washing with PBS, the cells were photographed under the microscope. To calculate the percentage of BrdU-positive cells, 10 fields $(\times 400)$ were chosen randomly and the percentage of BrdU-positive cells was calculated and expressed as means \pm S.D.

\section{Flow cytometry analysis}

To identify the quantity of CD34-positive cells among freshly isolated MNCs and to evaluate the development of surface markers on the cultured cells, flow cytometry analysis was performed on freshly isolated MNCs and on the cells cultured for 4, 14 and 28 days.

Briefly, the freshly isolated MNCs were washed twice with PBS, resuspended with PBS and then incubated at $4{ }^{\circ} \mathrm{C}$ for $30 \mathrm{~min}$ with directly conjugated primary murine monoclonal antibodies to CD34FITC (Becton-Dickinson). Fluorescent isotype-matched antibodies (Becton-Dickinson) were used as negative controls. The cells were then washed twice with PBS and resuspended in PBS. Quantitative fluorescence analysis was performed with a FACS-Calibur instrument and Cellquest Software (Becton-Dickinson).

The cells cultured for 4, 14 and 28 days were detached with 2 mM EDTA in PBS, treated with human IgG polyclonal antibody and washed with PBS. They were then incubated, as described above, with primary monoclonal antibodies to KDR,VE-cadherin, E-selectin (Santa Cruz) and vWF (Dako) as endothelial markers, and antibodies to CD14 (Becton-Dickinson) as monocytic markers. Isotype-matched antibodies were used as negative controls. For the detection of VWF and KDR, the cells were permeabilized with $0.1 \%$ Triton X-100 before incubation with the antibody. Fluorescent-conjugated secondary antibody (Becton-Dickinson) was added and the cells were incubated for $30 \mathrm{~min}$ at $4{ }^{\circ} \mathrm{C}$. After two washes in PBS, the cells were resuspended in PBS. Quantitative fluorescence analysis was then performed.

Three samples from different individuals were used in this test. The results are presented as means \pm S.D.

\section{Endothelial function assay}

eNOS mRNA expression The expression of eNOS in EPCs cultured for 4, 14 and 28 days was determined by reverse transcription-polymerase chain reaction (RT-PCR). The cells were rinsed twice in $1 \times$ PBS and total RNA was extracted using Trizol reagent (Invitrogen) according to the manufacturer's instructions. The cDNA was generated from $2 \mu \mathrm{g}$ of total RNA using MMLV reverse transcriptase (Promega) and oligo-d(T $)_{18}$ during a 2-h incubation at $42{ }^{\circ} \mathrm{C}$. PCR was performed with $2 \mu 1$ of cDNA using Taq DNA polymerase (Promega). Preliminary experiments were carried out to select the optimal number of cycles to enable the amplification reaction to proceed in a linear range. PCR of a constitutively expressed gene, GAPDH, was used as an internal control for the amount of input RNA. Initial cDNA denaturation was performed at $94{ }^{\circ} \mathrm{C}$ for $5 \mathrm{~min}$, followed by 35 cycles of denaturation at $94{ }^{\circ} \mathrm{C}$ for $30 \mathrm{~s}$, annealing at $58{ }^{\circ} \mathrm{C}$ for $30 \mathrm{~s}$, extension at $72{ }^{\circ} \mathrm{C}$ for $45 \mathrm{~s}$ and 
final extension at $72{ }^{\circ} \mathrm{C}$ for $5 \mathrm{~min}$. Human eNOS primers, $5^{\prime}$-GGC TCC CTC CTT CCG GCT-3' G upstream and 5'-TCC CGC AGC ACG CCG AT-3' downstream, generated a 468 bp product [21]. PCR assays were performed in triplicate. Control reactions were carried out using the GAPDH primers and the same templates. The PCR products were separated by electrophoresis on a $2 \%$ agarose gel with $1 \times$ TAE buffer, stained with EtBr and photographed under ultraviolet light.

In vitro angiogenesis assay An in vitro angiogenesis assay kit (Chemicon) was used to examine the ability of the cells to undergo angiogenesis. ECMatrix ${ }^{\mathrm{TM}}$ solution and the diluent buffer were thawed on ice or in a $4{ }^{\circ} \mathrm{C}$ refrigerator overnight. A mixture of 100 $\mu 1$ of $10 \times$ diluent buffer and $900 \mu$ of ECMatrix ${ }^{\mathrm{TM}}$ solution in a sterile microcentrifuge tube was prepared and kept on ice to avoid solidification. Fifty microliter of the mixture was transferred into a pre-cooled 96-well tissue culture plate. The plate was pre-incubated at $37^{\circ} \mathrm{C}$ for at least $1 \mathrm{~h}$ to allow the matrix solution to solidify. A total of $5 \times 10^{4}$ cells in $150 \mu$ of EGM- 2 medium were added into the wells and human umbilical vein-derived endothelial cells (HUVECs) were placed in other wells as control. Using a phase-control microscope from 5 to $24 \mathrm{~h}$, the adherent cells cultured for 4,14 and 28 days were assayed.

Monocyte/macrophage function assay-Dil-Ac-LDL and Indian ink double-uptake test

The cells were cultured in the medium supplemented with 10 $\mu \mathrm{g} / \mathrm{ml}$ of Dil labeled acetylated low-density lipoprotein (Dil-AcLDL) for $4 \mathrm{~h}$. The medium with Dil-Ac-LDL was washed away with PBS and medium with Indian ink $(10 \mu \mathrm{l} / \mathrm{ml})$ was added to the culture plate, which was then incubated for another $4 \mathrm{~h}$. The cells were observed with the phase-control fluorescent microscope. The cells cultured for 4,14 and 28 days were tested.

\section{Results}

\section{Morphology of adult EPCs}

Most of the MNCs seeded onto the plates were washed away by medium changes and only few of the small round cells were slightly attached to the bottom of the plates. After 2 to 3 days of culture, some of the attached cells elongated and became spindle-shaped. Around day 10 of culture, most of the attached cells had become spindleshaped, and at about day 20, the spindle-shaped cells started to become disc-shaped. These disc-like cells have been called mature ECs by many researchers (Figure 1). These cells rarely formed clusters and the proliferative capacity of the clusters did not last long. During continuous follow-up, the number of cells in the cluster did not increase significantly (Figure 2). Although the cells were similar to ECs morphologically, they were isolated and never reached complete confluence.
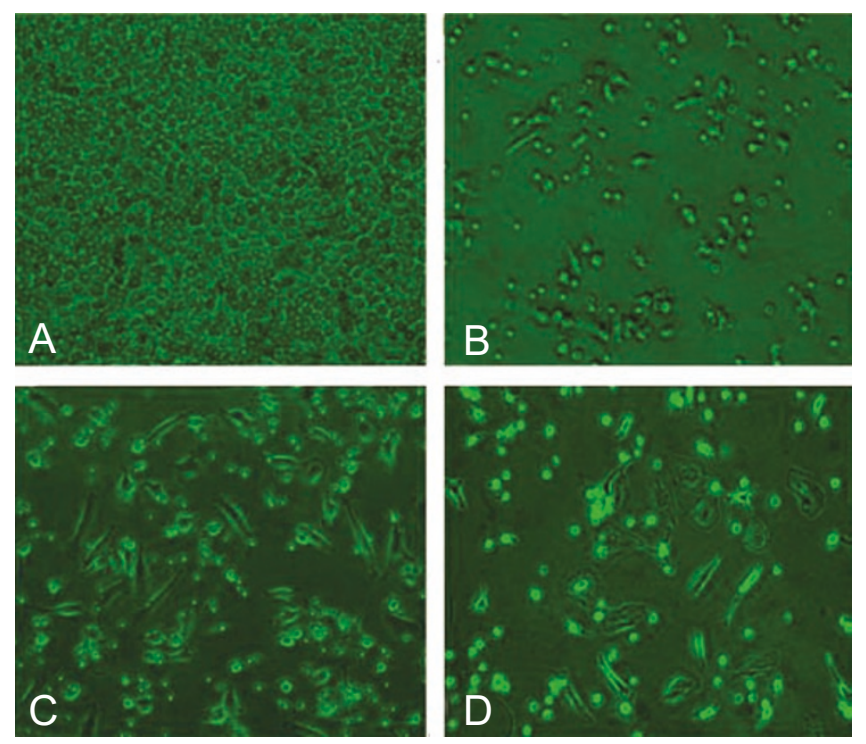

Figure 1 Evolution of the morphology of EPCs $(\times 200)$. (A) The MNCs plated on a culture dish. (B) The attached cells after the first medium change on day 3. (C) Most of the attached cells became spindle-shaped on day 10 of culture. (D) The spindle-shaped EPCs had become large disc-like cells by day 20 of culture.

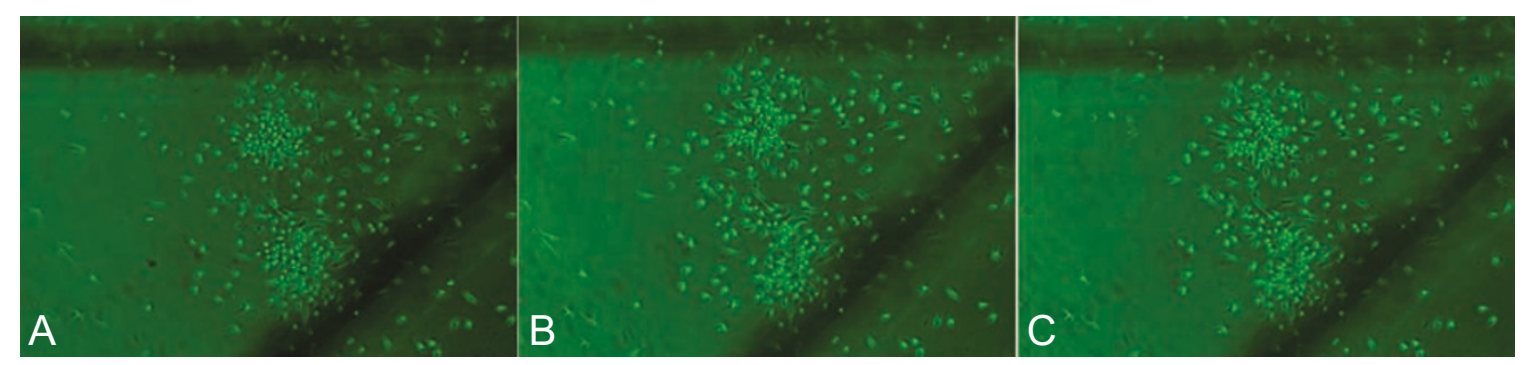

Figure 2 Continuous follow-up of a cell colony. The shadow in this view is a marker the author made to identify the same field (A: day 21; B: day 22; C: day 23) ( $\times 100)$. The panel demonstrates that the cell colony showed no significant change in cell number during three consecutive days. 
Proliferative capacity assay: growth curve

Since the cells never reached complete confluence, the growth curve of the primary cells reflected their proliferative capacity. At the early stage of culture, some of the slightly attached cells were washed away with the medium. Therefore, although the spindle-shaped cells showed some proliferative capacity, the total number of attached cells did not show a significant increase. At the late stage of culture, the decrease in cell number could be mainly attributed to cell death. Therefore, the growth curve of the cells showed a general downward trend (Figure 3 ).

\section{Proliferative capacity assay: BrdU incorporation assay}

We found that approximately $6.1 \% \pm 2.4 \%$ of the cells were positive for BrdU incorporation at day 7 , and most of the positive cells appeared at the center of the cells clusters (Figure 4A). At day 21, all the cells were negative for BrdU incorporation (Figure 4B), which indicates that the cells at the early stage had only a weak ability to proliferate, but the cells at the late stage stopped proliferating.

\section{Phenotype of the MNCs and the cultured cells}

We found that $0.39 \%$ of the MNCs from peripheral blood were positive for CD34 (Figure 5A), which means that freshly isolated MNCs contain the cells from which the EPCs originated. During in vitro culture, these cells demonstrated expression of the endothelial markers KDR, VE-cadherin and E-selectin. The percentage of the cells positive for KDR, VE-cadherin and E-selectin significantly increased in the earlier culture (from days 4 to 14 ) and remained stable at the late stage from days 14 to 28), However, the percentage of cells positive for vWF was very small and did not increase during the in vitro culture. Meanwhile, the cultured cells exhibited a

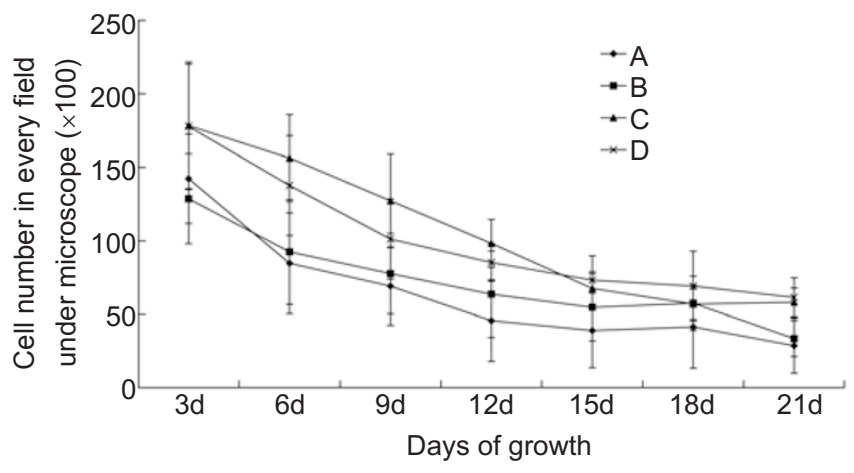

Figure 3 Growth curve of primary EPCs (A, B, C and D represent EPCs from different individuals) shows that the cells had no significant proliferative capacity.
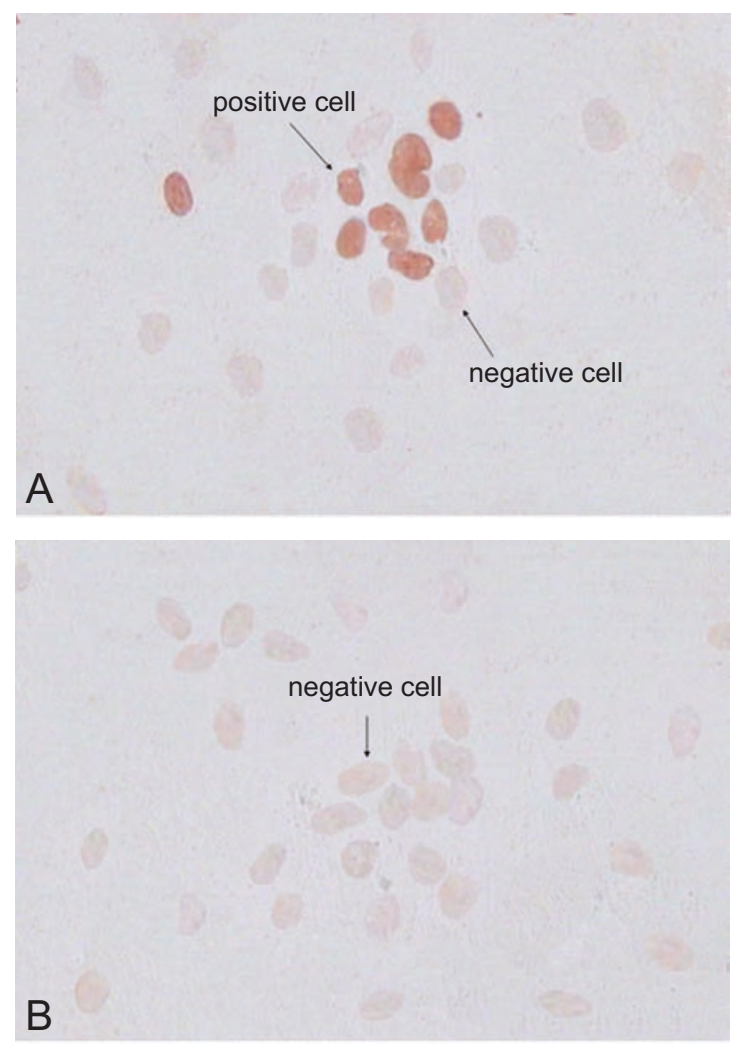

Figure 4 BrdU incorporation assay. (A) BrdU was incorporated into the nucleus of the cells cultured for 7 days. However, only $6.1 \% \pm 2.4 \%$ of the cells were positive for BrdU incorporation and most of them appeared in the center of the cell clusters. (B) At day 21 , all the cells became negative for BrdU incorporation $(\times 400)$.

high level of expression for the monocyte-specific marker, CD14, which remained stable throughout the culture (Figure 5B).

\section{Endothelial function assay}

To test the endothelial function of EPCs, RT-PCR analysis for the expression of eNOS and an in vitro angiogenesis assay were carried out. The expression level of eNOS in these cells was significantly lower than that of mature ECs derived from human umbilical vein as positive control (Figure 6). The cells cultured for 4, 14 and 28 days were also subjected to an in vitro angiogenesis assay. The results showed that the EPCs presented no sign of tube-like structure formation regardless of culture time, in contrast to the mature ECs from human umbilical vein (Figure 7).

\section{Monocyte/macrophage function assay for EPCs}

We performed the Dil-Ac-LDL and Indian ink doubleuptake test on days 4, 14 and 28 of culture. All the cells that 
A

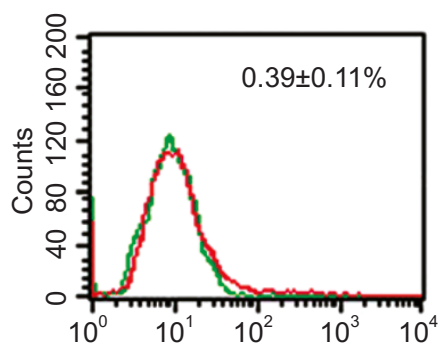

B
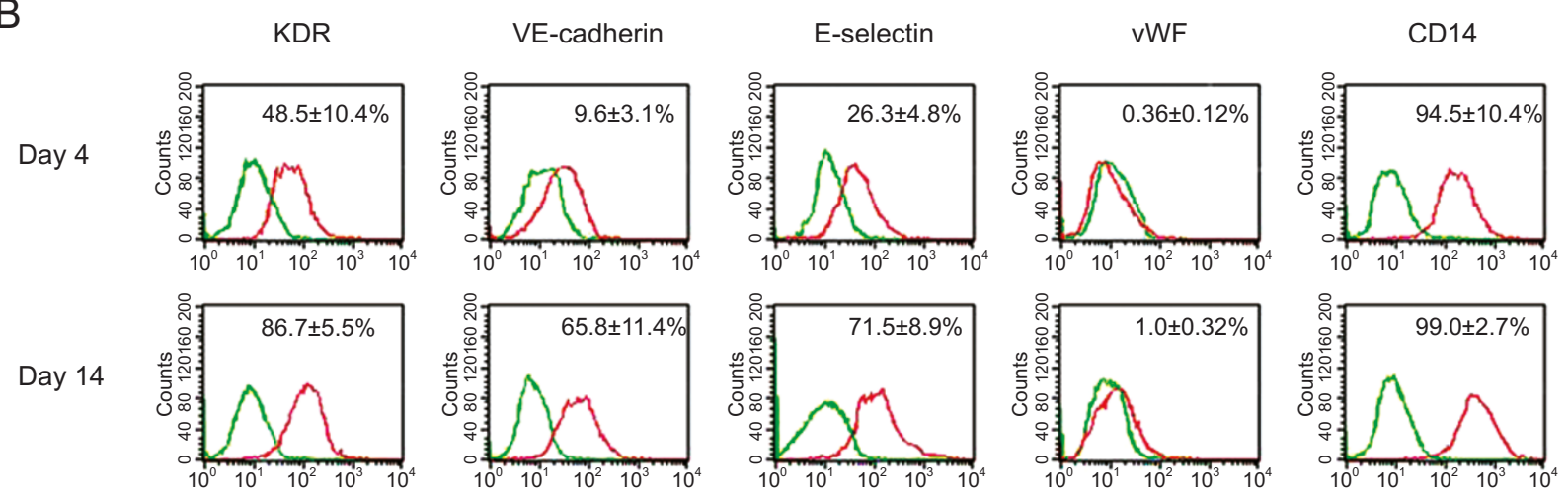

Day 28
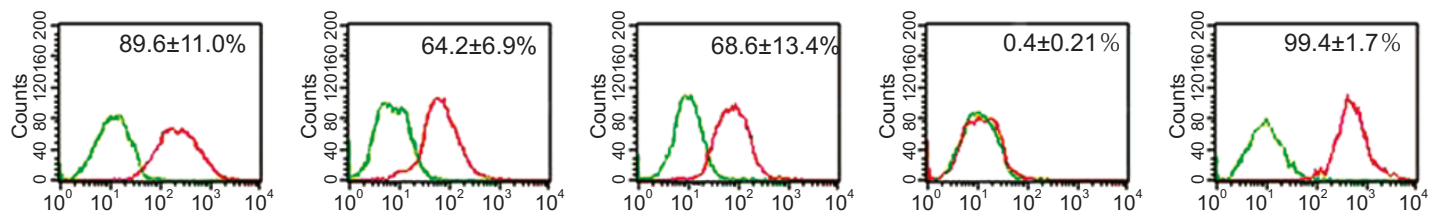

Figure 5 Flow cytometry analysis for phenotype of the MNCs and the cultured cells. (A) About $0.39 \%$ of the MNCs obtained by density gradient centrifugation were positive for CD34. (B) Expression of endothelial and monocyte markers on cultured cells (days 4, 14 and 28) were assessed. Similar results were obtained in three separate experiments. Numbers shown in the figure are the mean percent of the cells for the three results. Corresponding negative isotype controls are shown in green.

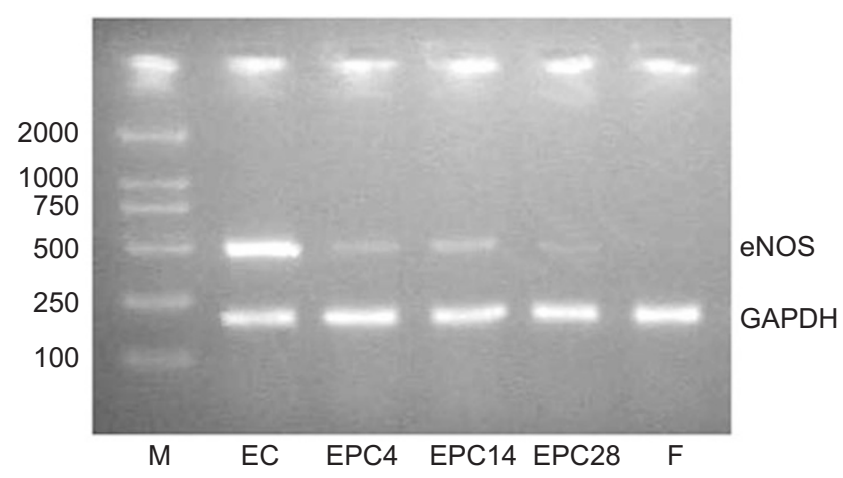

Figure 6 The expression of eNOS in EPCs. The expression level of eNOS in EPCs was much lower compared with that of mature endothelial cells from human umbilical vein. M: DNA marker, DL2000 (Takara); EC: human umbilical vein-derived endothelial cell; EPC4: EPCs cultured for 4 days; EPC14: EPCs cultured for 14 days; EPC28: EPCs cultured for 28 days; F: fibrocyte; eNOS: e-type NO synthesizer; GAPDH: glyceraldehyde-3-phosphatedehydrogenase.
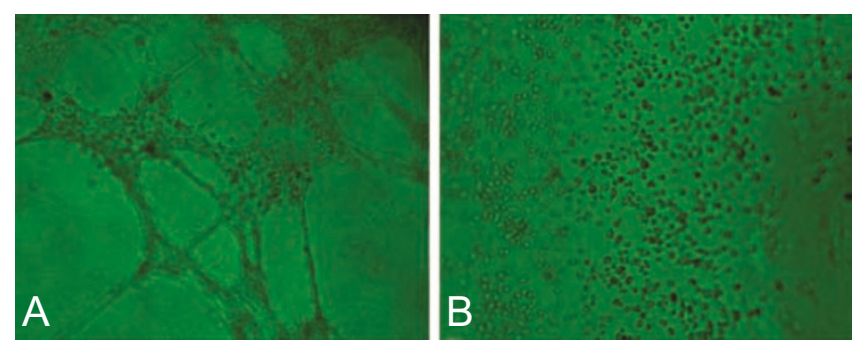

Figure 7 Comparison of the ability to form tube-like structure between HUVECs and EPCs by in vitro angiogenesis assay. (A) Tube-like structure formation in HUVECs as positive control $(\times$ 100). (B) No tube-like structure formation occurred in adult EPCs $(\times 100)$.

were positive for Dil-Ac-LDL uptake were also positive for uptake of Indian ink, indicating that they maintained their monocyte/macrophage function throughout the in 


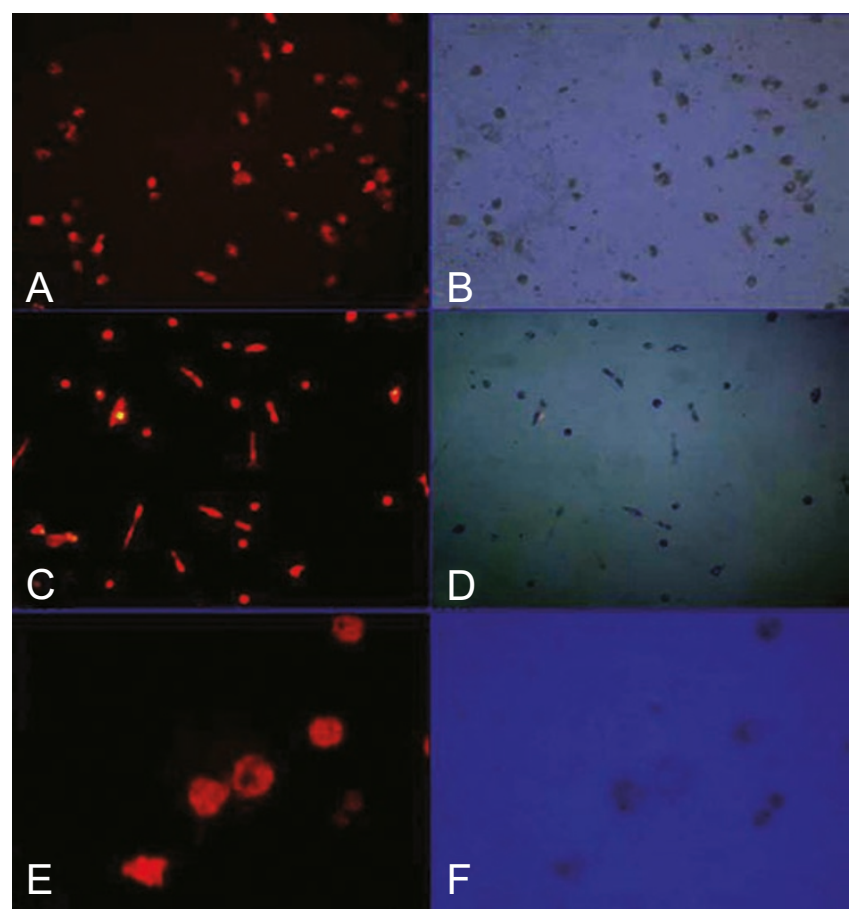

Figure 8 Dil-Ac-LDL and Indian ink double-uptake test. The cells positive for Dil-Ac-LDL uptake were also positive for Indian ink uptake. Afterwards, the cells maintained this function throughout the culture. $(\mathbf{A}, \mathbf{C}(\times 100)$ and $\mathbf{E}(\times 200))$ show the uptake of Dil-AcLDL. (B, D $(\times 100)$ and $\mathbf{F}(\times 200))$ show the uptake of Indian ink. A and $\mathbf{B}, \mathbf{C}$ and $\mathbf{D}$, and $\mathbf{E}$ and $\mathbf{F}$ show the cells from the same field, on days 4,14 and 28 of culture, respectively.

vitro culture (Figure 8).

\section{Discussion}

In our study, we obtained attached spindle-shaped cells that have been regarded as EPCs by some researchers [3]. However, these cells rarely developed clusters, and the proliferative capacity of the clusters did not last for a long time. The cells in the clusters never crowded together like ECs, regardless of how many MNCs were seeded onto the culture dishes. The adherent cells never reached complete confluence as described by Nakul-Aquaronne et al. [14].

Although the cells showed expression of some EC markers, such as KDR, VE-cadherin and E-selectin, only an extremely small percentage of the cells were positive for vWF. Also, the cells expressed CD14, which is the common antigen of monocytes. Therefore, cells obtained by this method can coexpress both endothelial and monocyte markers, similar to the properties of the CD34-positive cells described by Nakul-Aquaronne et al. [14]. In fact, before the discovery of EPCs, it had been reported that spindle-shaped cells derived from human peripheral MNCs can coexpress endothelial surface markers and monocytic markers in vitro [22]. Baroni et al. [23] and Buckley et al. [24] demonstrated that there is a special subset of monocyte/ macrophages that can express endothelial-related antigen in human lymphoid tissue. Therefore, we believe that the so-called EPCs obtained by in vitro culture of MNCs are monocytes derived from CD34-positive hematopoietic cells, which can express some endothelial characteristics. However, due to the effects of various growth factors, the expression of surface markers becomes uncertain, which may serve as an explanation why different groups have reported different surface marker profiles of EPCs [25]. Although the surface marker expression assay is the most frequently used method for the identification of cell type, determining the phenotype of cells by functional tests should be more persuasive.

To test the EC function possessed by EPCs, we examined the expression of eNOS, which has been reported to reflect the angiogenesis-promoting function of EPCs [3, 13, 17]. In our study, although the cells showed expression of eNOS, the expression level was much lower than that in HUVECs, which suggests that the cells obtained by this way are different from mature ECs. The in vitro angiogenesis assay, another commonly used test for EC function analysis, may reflect the capacity of ECs to participate in vasculogenesis. Endothelial cells from human umbilical vein (HUVECs) formed tube-like structures, in contrast to EPCs that failed to produce any structure. This may serve as further evidence that EPCs are functionally different from mature ECs in vitro. Several groups have reported that EPC-derived cells cannot function as mature ECs. Paunescu et al. [26] documented that EPCs from human umbilical cord blood differed functionally from HUVECs, as the former show a different reaction to TNF compared with mature ECs. Another study demonstrated that bone marrow-derived cells may not be incorporated into the vessel wall in vivo but only promote angiogenesis by excretion of certain factors, such as FGF-2, VEGF and MCP-1 [27]. In the present study, the functional test not only proved that the cells obtained by culture of MNCs could not function as mature ECs but also gave strong evidence that the cells maintained monocyte function.

In our study, for the first time, we performed a phagocytosis function test and followed up the evolution of this function. It is well known that Indian ink uptake is a test used to evaluate phagocytic function of leukocytes/ monocytes [28] and LDL uptake is a method used to test the function of both monocytes and ECs. Therefore, the combination of the Indian ink uptake and LDL uptake tests does not only show phagocytic function of the cells but can also be used to demonstrate the existence of "true EPCs" [29] without monocyte/macrophage characteristics. 
Our results showed that cells that are positive for LDL uptake can also take up Indian ink. It is suggested that the EPCs obtained in this manner maintain monocyte/ macrophage function throughout in vitro culture. Hur et al. [17] reported a subpopulation of EPCs designated as "late EPCs", which present typical ECs characteristics and a strong proliferative capacity in peripheral blood, which may serve as evidence for the existence of "true EPCs". However, in the present study, we did not find any EPCs with significant proliferative capacity. As ECs can slough off from the vessel wall and some of the ECs from the aorta have a stronger proliferative capacity than was supposed [30], it is difficult to preclude that the "late EPCs" are the ECs sloughed off from the blood vessel wall, which show strong proliferation under EC growth-promoting conditions. Identification of "true EPCs" among bone marrow cells with more EPCs and less contamination of peripheral blood should be more persuasive in confirming the existence of "true EPCs".

In conclusion, although several subsets of EPCs in human have been reported [29], in this study, by culture of MNCs from human peripheral blood, only one subset of "EPCs" with both endothelial and monocyte characteristics was obtained. However, these cells maintained phagocytic function throughout in vitro culture, and showed no strong proliferative capacity. We failed to identify "true EPCs" and further studies are necessary to prove their existence.

\section{Acknowledgments}

This work was supported by the National Natural Science Foundation of China (Nos. 30170932 and 30371411) and the Foundation for Excellent Young Scholar (No. 30125039).

\section{References}

1 Risau W, Sariola H, Zerwes HG, et al. Vasculogenesis and angiogenesis in embryonic-stem-cell-derived embryoid bodies. Development 1988; 102:471-478.

2 Pardanaud L, Yassine F, Dieterlen-Lievre F. Relationship between vasculogenesis, angiogenesis and haemopoiesis during avian ontogeny. Development 1989; 105:473-485.

3 Asahara T, Murohara T, Sullivan A, et al. Isolation of putative progenitor endothelial cells for angiogenesis. Science 1997; 275:964-967.

4 Kalka C, Masuda H, Takahashi T, et al. Transplantation of ex vivo expanded endothelial progenitor cells for therapeutic neovascularization. Proc Natl Acad Sci USA 2000; 97:34223427.

5 Murohara T, Ikeda H, Duan J, et al. Transplanted cord blood-derived endothelial precursor cells augment postnatal neovascularization. J Clin Invest 2000; 105:1527-1536.

6 Kawamoto A, Gwon HC, Iwaguro H, et al. Therapeutic potential of ex vivo expanded endothelial progenitor cells for myocardial ischemia. Circulation 2001; 103:634-637.

7 Assmus B, Schachinger V, Teupe C, et al. Transplantation of progenitor cells and regeneration enhancement in acute myocardial infarction (TOPCARE-AMI). Circulation 2002; 106:3009-3017.

8 Kawamoto A, Tkebuchava T, Yamaguchi J, et al. Intramyocardial transplantation of autologous endothelial progenitor cells for therapeutic neovascularization of myocardial ischemia. Circulation 2003; 107:461-468.

9 Werner N, Junk S, Laufs U, et al. Intravenous transfusion of endothelial progenitor cells reduces neointima formation after vascular injury. Circ Res 2003; 93:e17-e24.

10 Britten MB, Abolmaali ND, Assmus B, et al. Infarct remodeling after intracoronary progenitor cell treatment in patients with acute myocardial infarction (TOPCARE-AMI): mechanistic insights from serial contrast-enhanced magnetic resonance imaging. Circulation 2003; 108:2212-2218.

11 Shirota T, He H, Yasui H, Matsuda T. Human endothelial progenitor cell-seeded hybrid graft: proliferative and antithrombogenic potentials in vitro and fabrication processing. Tissue Eng 2003; 9:127-136.

12 Griese DP, Ehsan A, Melo LG, et al. Isolation and transplantation of autologous circulating endothelial cells into denuded vessels and prosthetic grafts: implications for cell-based vascular therapy. Circulation 2003; 108:2710-2715.

13 Harraz M, Jiao C, Hanlon HD, Hartley RS, Schatteman GC. CD34- blood-derived human endothelial cell progenitors. Stem Cells 2001; 19:304-312.

14 Nakul-Aquaronne D, Bayle J, Frelin C. Coexpression of endothelial markers and CD14 by cytokine mobilized CD34+ cells under angiogenic stimulation. Cardiovasc Res 2003; 57:816823.

15 Lin Y, Weisdorf DJ, Solovey A, Hebbel RP. Origins of circulating endothelial cells and endothelial outgrowth from blood. J Clin Invest 2000; 105:71-77.

16 Gulati R, Jevremovic D, Peterson TE, et al. Diverse origin and function of cells with endothelial phenotype obtained from adult human blood. Circ Res 2003; 93:1023-1025.

17 Hur J, Yoon CH, Kim HS, et al. Characterization of two types of endothelial progenitor cells and their different contributions to neovasculogenesis. Arterioscler Thromb Vasc Biol 2004; 24:288-293.

18 Tepper OM, Galiano RD, Capla JM, et al. Human endothelial progenitor cells from type II diabetics exhibit impaired proliferation, adhesion, and incorporation into vascular structures. Circulation 2002; 106:2781-2786.

19 Assmus B, Urbich C, Aicher A, et al. HMG-CoA reductase inhibitors reduce senescence and increase proliferation of endothelial progenitor cells via regulation of cell cycle regulatory genes. Circ Res 2003; 92:1049-1055.

20 Verma S, Kuliszewski MA, Li SH, et al. C-reactive protein attenuates endothelial progenitor cell survival, differentiation, and function: further evidence of a mechanistic link between C-reactive protein and cardiovascular disease. Circulation 2004; 109:2058-2067.

21 Nakai K, Fujii S, Yamamoto A, et al. Effects of high glucose on NO synthesis in human keratinocyte cell line (HaCaT). J Dermatol Sci. 2003; 31:211-218.

22 Browning PJ, Sechler JM, Kaplan M, et al. Identification and 
culture of Kaposi's sarcoma-like spindle cells from the peripheral blood of human immunodeficiency virus-1-infected individuals and normal controls. Blood 1994; 84:2711-2720.

23 Baroni CD, Vitolo D, Remotti D, et al. Immunohistochemical heterogeneity of macrophage subpopulations in human lymphoid tissues. Histopathology 1987; 11:1029-1042.

24 Buckley PJ, Dickson SA, Walker WS. Human splenic sinusoidal lining cells express antigens associated with monocytes, macrophages, endothelial cells, and T lymphocytes. J Immunol 1985; 134:2310-2315.

25 Walenta K, Friedrich EB, Sehnert F, Werner N, Nickenig G. In vitro differentiation characteristics of cultured human mononuclear cells - implications for endothelial progenitor cell biology. Biochem Biophys Res Commun 2005; 333:476-482.

26 Paunescu V, Suciu E, Tatu C, et al. Endothelial cells from hematopoietic stem cells are functionally different from those of human umbilical vein. J Cell Mol Med 2003; 7:455-460.
27 Ziegelhoeffer T, Fernandez B, Kostin S, et al. Bone marrowderived cells do not incorporate into the adult growing vasculature. Circ Res 2004; 94:230-238.

28 Muftuoglu TM, Koksal N, Ozkutlu D. Evaluation of phagocytic function of macrophages in rats after partial splenectomy. J Am Coll Surg 2000; 191:668-671.

29 Rookmaaker MB, Vergeer M, van Zonneveld AJ, Rabelink TJ, Verhaar MC. Endothelial progenitor cells: mainly derived from the monocyte/macrophage-containing CD34- mononuclear cell population and only in part from the hematopoietic stem cellcontaining CD34+ mononuclear cell population. Circulation 2003; 108:e150.

30 Ingram DA, Mead LE, Moore DB, et al. Vessel wall-derived endothelial cells rapidly proliferate because they contain a complete hierarchy of endothelial progenitor cells. Blood. 2005; 105:2783-2786.

Edited by Jacques Samarut 